\title{
In-orbit performance of the Herschel/SPIRE imaging Fourier transform spectrometer: lessons learned
}

\author{
David A. Naylor ${ }^{*}$, Jean-Paul Baluteau ${ }^{b}$, George J. Bendo ${ }^{c}$, Dominique Benielli ${ }^{b}$, Trevor R. \\ Fulton $^{a, d}$, Brad G. Gom ${ }^{a}$, Matthew J. Griffin ${ }^{e}$, Rosalind Hopwood ${ }^{f}$, Peter Imhof ${ }^{a, d}$, Tanya L. Lim ${ }^{g}$, \\ Nanyao Lu ${ }^{h}$, Gibion Makiwa ${ }^{a}$, Nicola Marchili ${ }^{i}$, Glenn S. Orton ${ }^{j}$, Andreas Papageorgiou ${ }^{e}$, Chris \\ Pearson $^{g, k}$, Edward T. Polehampton ${ }^{a, g}$, Bernhard Schulz ${ }^{h}$, Locke D. Spencer ${ }^{a}$, Bruce M. Swinyard $^{l, g}$, \\ Ivan Valtchanov ${ }^{m}$, Matthijs H. D. van der Wiel ${ }^{a}$, Ian T. Veenendaal ${ }^{a}$, Ronin $\mathrm{Wu}^{n}$ \\ ${ }^{a}$ Institute for Space Imaging Science, University of Lethbridge, Alberta, T1K 3M4, Canada \\ ${ }^{b}$ Laboratoire d'Astrophysique de Marseille, Marseille, France, 13388 \\ ${ }^{c}$ UK ALMA Regional Centre Node, University of Manchester, M13 9PL, UK \\ ${ }^{d}$ Blue Sky Spectroscopy Inc., 9 - 7404 Avenue South, Lethbridge, Alberta, T1J 0N9, Canada \\ ${ }^{e}$ School of Physics and Astronomy, Cardiff University, Cardiff, United Kingdom, CF24 3AA, UK \\ fDepartment of Physics, Imperial College London, SW7 2AZ, UK \\ ${ }^{g}$ RAL Space, Rutherford Appleton Laboratory, Chilton, Didcot, OX11 0QX, UK \\ ${ }^{h}$ NHSC/IPAC, California Institute of Technology, Pasadena, CA 91125, USA \\ ${ }^{i}$ Dipartimento di Fisica e Astronomia, Università di Padova, I-35131 Padova, Italy \\ jJet Propulsion Laboratory, California Institute of Technology, Pasadena, CA 91109, USA \\ ${ }^{k}$ Department of Physical Sciences, The Open University, MK7 6AA, UK \\ ${ }^{l}$ Department of Physics and Astronomy, University College London, UK \\ ${ }^{m}$ Herschel Science Centre, ESAC, ESA, E-28691 Villanueva de la Cañada, Spain \\ ${ }^{n}$ Laboratoire AIM, CEA Saclay, F-91191 Gif-sur-Yvette, France
}

\begin{abstract}
The Spectral and Photometric Imaging Receiver (SPIRE) is one of three scientific instruments on board the European Space Agency's Herschel Space Observatory which ended its operational phase on 29 April 2013. The low to medium resolution spectroscopic capability of SPIRE is provided by an imaging Fourier transform spectrometer (iFTS) of the Mach-Zehnder configuration. With their high throughput, broad spectral coverage, and variable resolution, coupled with their well-defined instrumental line shape and intrinsic wavelength and intensity calibration, iFTS are becoming increasingly common in far-infrared space astronomy missions. The performance of the SPIRE imaging spectrometer will be reviewed and example results presented. The lessons learned from the measured performance of the spectrometer as they apply to future missions will be discussed.
\end{abstract}

Keywords: Herschel, SPIRE, imaging Fourier transform spectrometer, performance

\section{INTRODUCTION}

The Spectral and photometric imaging receiver (SPIRE) is one of three scientific instruments aboard the European Space Agency's Herschel Space Observatory ${ }^{1}$ launched on 14 May 2009. The observational phase of the mission ended on 29 April 2013, following expiration of the onboard supply of liquid helium. SPIRE ${ }^{2}$ contains a three band imaging photometer operating at 250,350, and $500 \mu \mathrm{m}$, and an imaging Fourier transform spectrometer (iFTS), which covers the wavelength range of 194-671 $\mu \mathrm{m}$ (447-1544 GHz; 14.9-51.5 $\left.\mathrm{cm}^{-1}\right)$. The SPIRE instrument, shown in Figure 1, is approximately $700 \times 400 \times 400 \mathrm{~mm}$ in size and is supported on the $\sim 4 \mathrm{~K}$ Herschel optical bench by thermally insulating mounts. The photometer and spectrometer are mounted on opposite sides of the SPIRE optical bench, which contains the optics, bolometer arrays (three for the photometer, and two for the spectrometer), an internal ${ }^{3}$ He cooler to provide the required detector operating temperature of $\sim 0.3 \mathrm{~K}$, filters, mechanisms, internal calibrators, and housekeeping

\footnotetext{
*David A. Naylor: Email: naylor@uleth.ca, Telephone: 14033292426
}

Space Telescopes and Instrumentation 2014: Optical, Infrared, and Millimeter Wave, edited by Jacobus M. Oschmann, Jr., Mark Clampin, Giovanni G. Fazio, Howard A. MacEwen, Proc. of SPIE Vol. 9143, 91432D · C 2014 SPIE CCC code: $0277-786 \mathrm{X} / 14 / \$ 18 \cdot$ doi: $10.1117 / 12.2054989$ 
thermometers. Both the photometer and spectrometer have cold pupil stops conjugate with the Herschel secondary mirror, which is the telescope system pupil, and defines an effective $3.29 \mathrm{~m}$ diameter primary aperture. All five bolometer arrays use hexagonally close-packed, conical feedhorn-coupled spider web neutron transmutation doped (NTD) Germanium bolometers. The bolometers are AC biased with frequency adjustable between 50 and $200 \mathrm{~Hz}$, avoiding $1 / \mathrm{f}$ noise from the cold JFET readout.

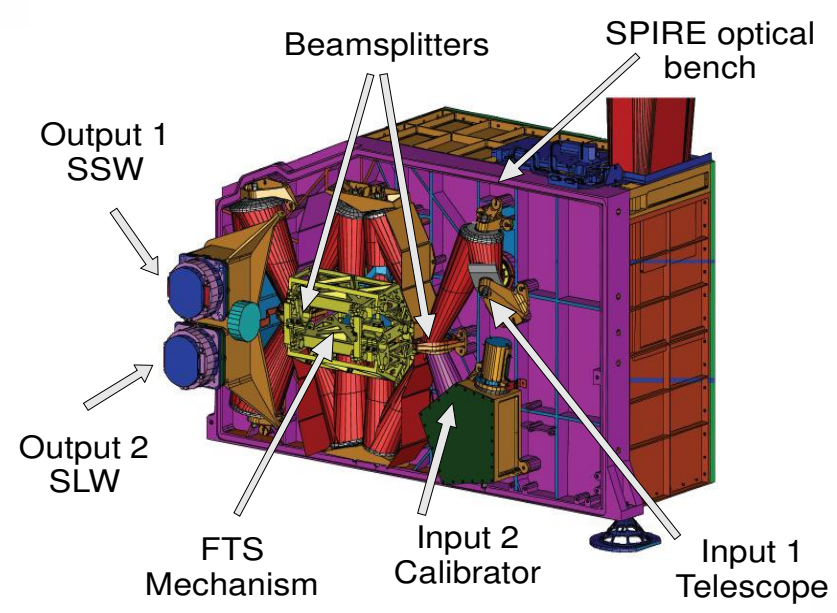

Figure 1. The Herschel/SPIRE MZ iFTS. Light from the Herschel telescope enters through input 1 . The temperature of the optical bench is $\sim 4 \mathrm{~K}$; the short and long wavelength bolometer arrays (SSW, SLW) are at $\sim 0.3 \mathrm{~K}$. Adapted from Griffin et al. ${ }^{3}$.

The spectrometer is of the Mach-Zehnder (MZ) configuration ${ }^{4}$ and uses two broadband intensity beamsplitters ${ }^{5}$ to provide spatial separation of the input and output ports. One input port views a 2.6 arcmin diameter unvignetted field of view (FOV) on the sky and the other an on-board reference source (SCAL). Two bolometer arrays at the output ports cover overlapping bands of 194-313 $\mu \mathrm{m}$ (SSW) and 303-671 $\mu \mathrm{m}$ (SLW). As with any FTS, each scan of the moving mirror produces an interferogram in which the spectrum of the entire band is encoded with the spectral resolution corresponding to the maximum mirror travel. Specifications of the SPIRE spectrometer are summarized in Table 1. A detailed description of the SPIRE spectrometer is given elsewhere ${ }^{2}$.

Table 1. Summary of the SPIRE iFTS instrument specifications.

\begin{tabular}{|c|c|}
\hline Spectral range & $14.9-51.5 \mathrm{~cm}^{-1} ; 447-1544 \mathrm{GHz} ; 671-194 \mu \mathrm{m}$ (section 2.1$)$ \\
\hline Maximum spectral resolution & $0.04 \mathrm{~cm}^{-1} ; \sigma / \Delta \sigma=384 @ 650 \mu \mathrm{m}, 1250 @ 200 \mu \mathrm{m}$ \\
\hline Spectral resolution modes & Low $\left(0.83 \mathrm{~cm}^{-1}\right)$, high $\left(0.04 \mathrm{~cm}^{-1}\right)$ \\
\hline Effective telescope diameter & $3.29 \mathrm{~m}$ \\
\hline Beam diameter at rooftop mirror & $\sim 20 \mathrm{~mm}$ \\
\hline Detector optics & Feedhorn coupled \\
\hline Beam shape & Gaussian and Multi-moded \\
\hline Angular resolution & $17-42$ arcsec (FWHM equivalent beam) \\
\hline Detector arrays & $37(\mathrm{SSW})+19$ (SLW) NTD Ge Bolometers \\
\hline Instantaneous unvignetted field of view & Circular, 2.6 arcmin diameter \\
\hline Imaging & $2 F \lambda$ spacing; Nyquist sampling with $4 \times 4$ jiggle pattern \\
\hline$\overline{\text { Size }}$ & $0.7 \mathrm{~m} \times 0.4 \mathrm{~m} \times 0.4 \mathrm{~m}$ \\
\hline Mass & $91 \mathrm{~kg}$ (including three-band photometer) \\
\hline Dominant noise source & $\sim 85 \mathrm{~K}$ primary telescope dish \\
\hline Operational temperature & $4.5 \mathrm{~K}$ (optical bench), $0.3 \mathrm{~K}$ (detectors), $\sim 85 \mathrm{~K}$ (primary mirror) \\
\hline NEFD ${ }^{\dagger}$ per bolometer $0.04 \mathrm{~cm}^{-1}$ & $\sim 1000 \mathrm{mJy}$ (5-sigma, 1 hour $)^{\ddagger}$ \\
\hline Spectral line sensitivity & $\sim 10^{-17} \mathrm{~W} / \mathrm{m}^{2}$ (5-sigma, 1 hour $)$ \\
\hline
\end{tabular}

\footnotetext{
Noise equivalent flux density.

A milliJansky (mJy) is a unit of flux density commonly used in astronomy; $1 \mathrm{Jy}=10^{-26} \mathrm{~W} \mathrm{~m}^{-2} \mathrm{~Hz}^{-1}$.
} 


\section{IN-FLIGHT PERFORMANCE}

Following a successful launch, the commissioning and performance verification (PV) phases of the mission revealed that all SPIRE subsystems were fully functional and met their key requirements ${ }^{6}$. In this paper we compare the performance of the SPIRE instrument as determined from the early mission data with that now realized during the post operations phase of the mission. The PV phase data were processed using earlier versions of the Herschel Data Processing System ${ }^{7}$; the current analysis is based on Herschel Interactive Processing Environment (HIPE) version 12. The enhanced data quality is due to a better understanding of the instrument and in particular improved calibration schema and products. These ongoing improvements, which are summarized in the following sections, are being conducted within the framework of the three year post-operational phase of Herschel/SPIRE, by a dedicated team working at several institutions. References are provided to allow the reader to study specific topics in greater detail.

\subsection{Spectral range}

The SPIRE iFTS was designed to cover the spectral range 447-1544 GHz to match the emission peak of continuum and line emission from the cold interstellar medium. This range is also covered by the Herschel/HIFI instrument ${ }^{8}$ and has a small overlap with the long wavelength end of the Herschel/PACS instrument ${ }^{9}$. The two bands of the SPIRE iFTS were designed to have a small overlap to simplify cross-calibration while maximizing the spectral range. The spectral bandpass supplied in the standard pipeline was initially chosen based on the half power points of the bolometer filter profiles, the high pass edges being determined by the exit apertures of the conical feedhorns, and the low pass edges determined by the optical filters placed immediately in front of the bolometer arrays. A reduced bandpass was adopted from HIPE v8 onwards to restrict noise spikes at the edges of the band. Subsequent improvements in calibration ${ }^{10-13}$ have meant that the effective bands can be widened for HIPE v12.1, especially at the high frequency ends. However, the narrower HIPE v8 range is maintained as the basis for the officially validated calibration ${ }^{13}$. The results are presented in Figure 2 and Table 2.
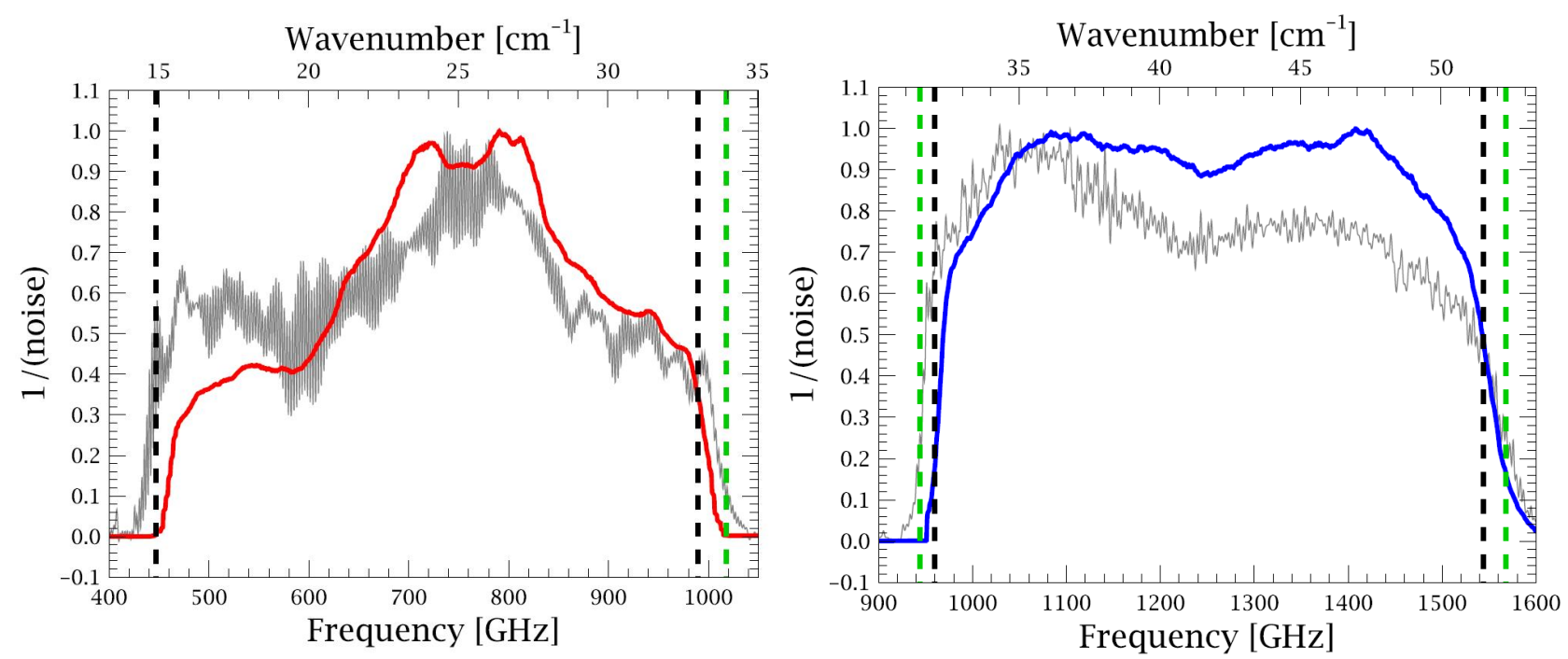

Figure 2. Spectral range of the central bolometers of each array (i.e., SLWC3 (left) and SSWD4 (right)), determined from in-flight measurements ${ }^{2}$. The normalized telescope RSRFs ${ }^{12}$ are shown in grey. The red and blue lines show the normalized reciprocal of the noise. The dashed lines indicate the bandpass limits for HIPE v8 (black) and the new extended HIPE v12.1 limits (green).

Table 2. Comparison of measured spectral ranges for the SLW and SSW bands.

\begin{tabular}{|l|l|l|}
\hline & \multicolumn{1}{|c|}{ SLW } & SSW \\
\hline Reported in [2] & $14.9-33.5 \mathrm{~cm}^{-1}(447-1004 \mathrm{GHz})$ & $31.5-51.8 \mathrm{~cm}^{-1}(944-1553 \mathrm{GHz})$ \\
\hline From HIPE v8 & $14.9-33.0 \mathrm{~cm}^{-1}(447-989 \mathrm{GHz})$ & $32.0-51.5 \mathrm{~cm}^{-1}(959-1544 \mathrm{GHz})$ \\
\hline From HIPE v12.1 & $14.9-34.0 \mathrm{~cm}^{-1}(447-1018 \mathrm{GHz})$ & $31.5-52.3 \mathrm{~cm}^{-1}(944-1568 \mathrm{GHz})$ \\
\hline
\end{tabular}




\subsection{Instrumental line shape (ILS)}

In the case of an ideal Fourier transform spectrometer, the instrumental line shape is given by the cardinal sine or sinc function:

$$
I L S(\sigma)=2 L \sin (2 \pi \sigma L) / 2 \pi \sigma L
$$

where $\mathrm{L}$ is the maximum optical path difference (OPD) created by the scan mirror travel and $\sigma$ is the wavenumber [cm ${ }^{-1}$ ]. The sinc function has a full width at half maximum (FWHM) of $\sim 0.603 / L$ and is characterized by a series of secondary lobes of slowly decreasing amplitude, the amplitude of the first minimum being $-21.7 \%$ of the main lobe. Using a technique known as apodization it is possible post hoc to decrease the amplitudes of the sidelobes associated with the sinc function at the cost of increasing the FWHM of the ILS (i.e., decreasing the spectral resolution) ${ }^{14}$. However, the accuracy of derived spectral line parameters is improved if all the information contained in the extended wings of the ILS is used in the subsequent data analysis.

The ILS can be determined from the 10 isolated CO lines that fall within the SPIRE iFTS band. The previously reported ILS $^{6}$ was determined from measurements of CO line emission originating from the Orion molecular cloud, which is an extended source $^{15}$. To investigate the potential impact of source extent on the ILS, measurements of CO line emission from point, semi-extended and extended sources were compared. Figure 3 shows measurements of two CO lines, which lie near the ends of the SLW and SSW spectral bands, for sources of varying extent. There appears to be little dependence between line shape and source extent. However, since point sources are expected to provide the best estimate of the ILS, the empirical line shape has been determined from observations of NGC7027, one of the routine calibration sources ${ }^{16}$.
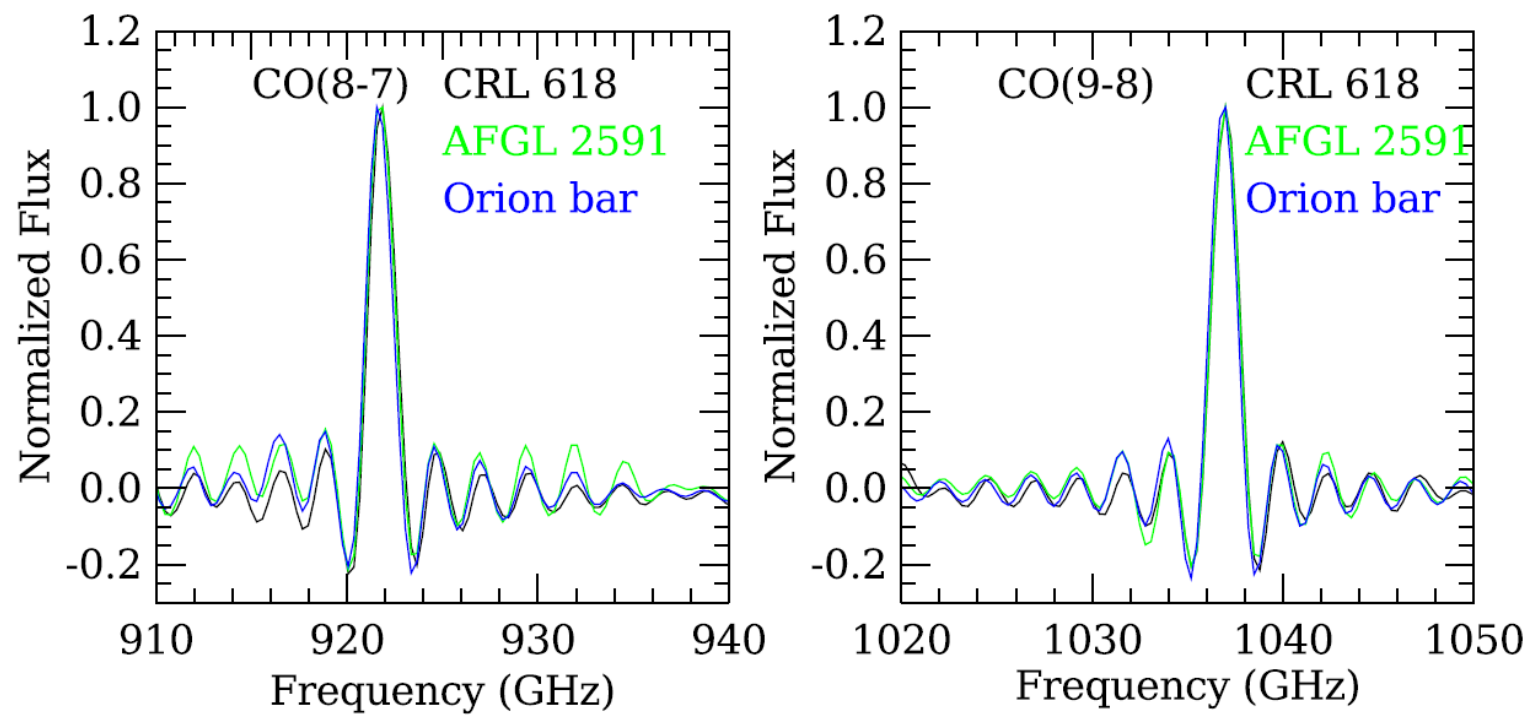

Figure 3. Normalized, continuum subtracted, spectra of the CO (8-7) and CO (9-8) lines that lie near the ends of the SLW and SSW bands, respectively, for point, semi-extended and extended sources (CRL618, AFGL 2591 and Orion bar, respectively).

In section 2.5 we show that the beam profile for the SSW band is significantly better defined than that of the SLW band. Since the beam profile impacts the ILS ${ }^{17}$, the empirical ILS has been derived from non-blended, unresolved, CO lines of a point-like source in the SSW band. Figure 4 shows the resulting ILS obtained from the average of the five CO lines

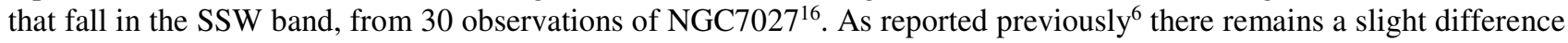
between the derived ILS and the classical sinc function, particularly in the region of the first negative high frequency lobe. While a small asymmetry in the ILS is to be expected from the effect of the finite entrance aperture of the spectrometer $^{17}$, it is possible that the residual asymmetry is due in part to a slight optical misalignment within the interferometer itself. Since advanced line fitting routines require a detailed knowledge of the ILS in order to retrieve 
accurate spectral line parameters (e.g. position, intensity, width), and to search for weak lines in the presence of strong ones, we continue to investigate the cause of the residual asymmetry.

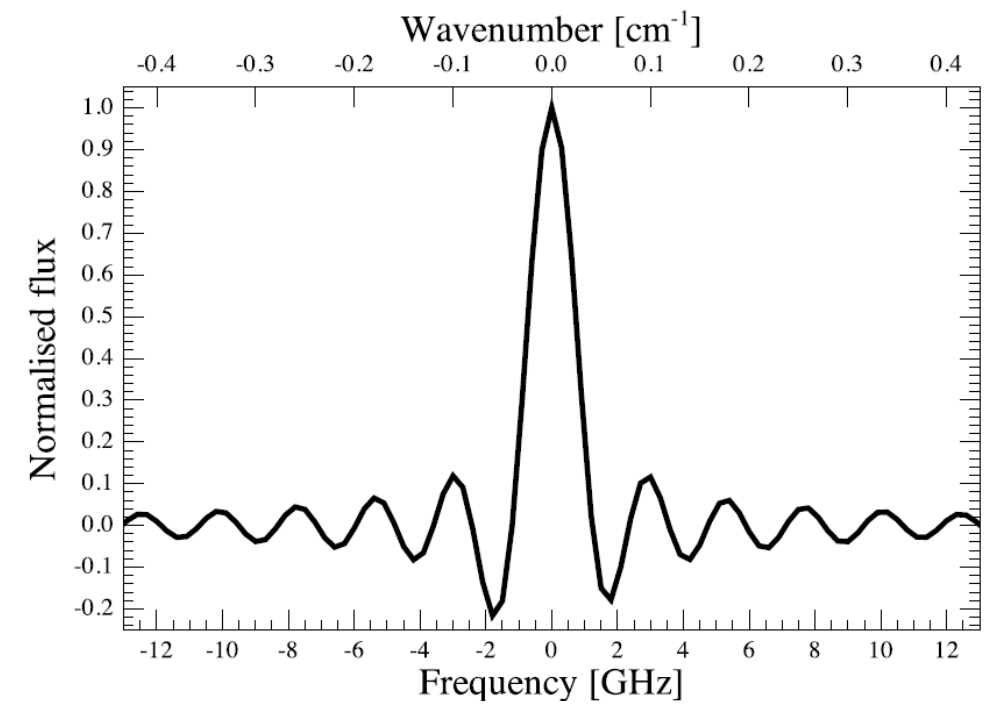

Figure 4. Empirical line shape derived from repeated observations of the CO lines in the SSW band from NGC7027.

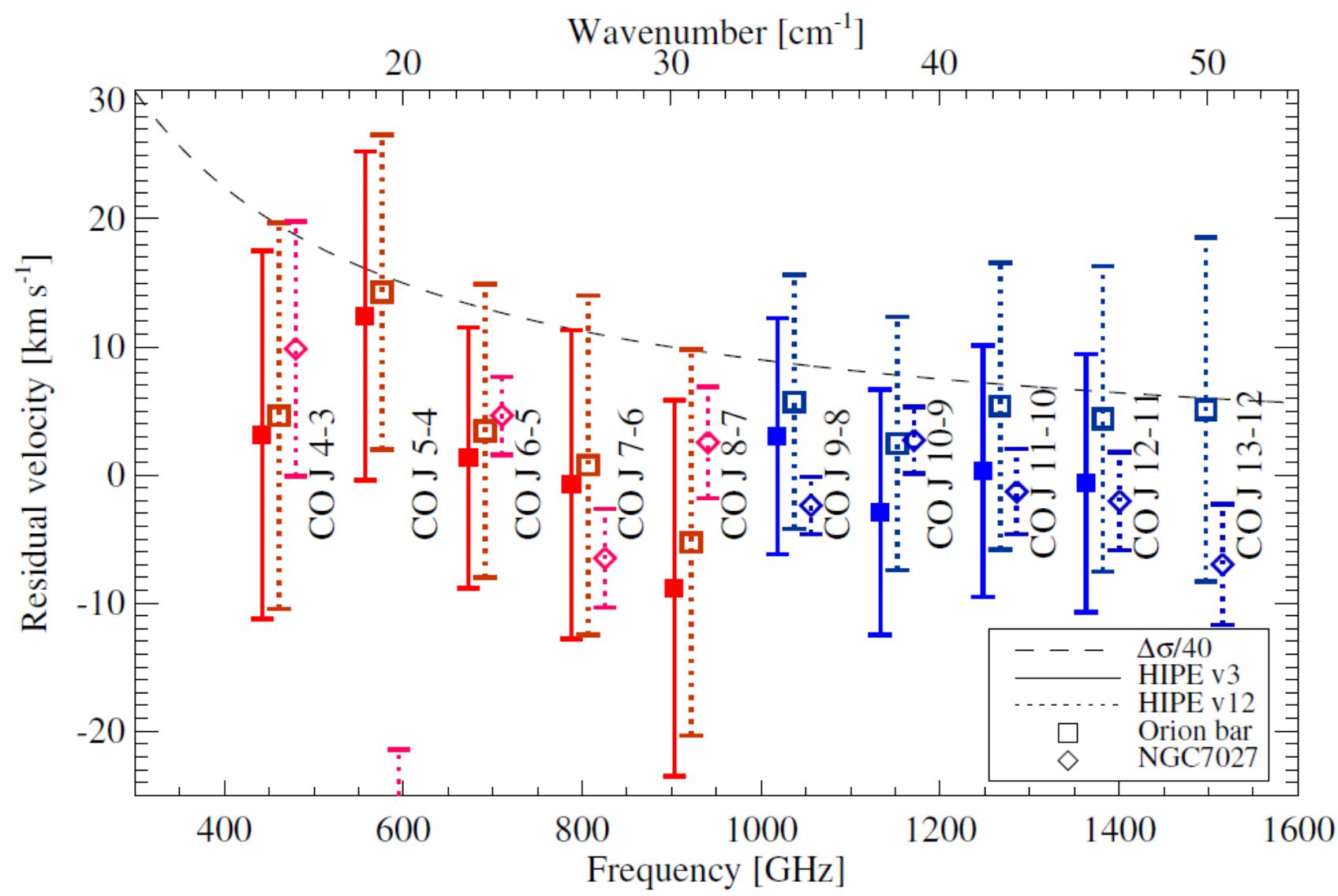

Figure 5. The wavelength accuracy expressed in terms of residual velocity, for CO lines observed in the point-like source NGC7027 (diamonds) and the extended Orion bar (squares). The solid lines and symbols represent the HIPE v3 results; the dotted lines and hollow symbols represent the latest HIPE v12 results. The dashed line corresponds to an uncertainty of $1 / 40^{\text {th }}$ of a resolution element. Horizontal offsets are introduced to visually separate each data set. 


\subsection{Wavelength accuracy}

For an infinitely small detector located on the optical axis of a Fourier transform spectrometer, the wavelength scale is uniquely determined by the metrology system. For detectors of finite size the scale must be corrected for the effects of obliquity $6,17,18$; the same is true for off-axis detectors. Since the SPIRE iFTS detector and metrology readout are measured at different times, but with respect to the same spacecraft master clock, an interpolation algorithm was necessary to produce the signal as a function of optical path difference within the interferometer ${ }^{19}$. The high signal-tonoise CO lines in the spectra of the point-like routine calibration source, NGC 7027, were used to confirm the wavelength accuracy expressed as a residual velocity uncertainty ${ }^{13}$. In determining the residual velocity, both the effects of obliquity and of Doppler motion of the source and Herschel telescope have been removed. In agreement with other practitioners ${ }^{17}$ we find that the line centres can be determined to within a small fraction of the spectral resolution element if the signal-to-noise is sufficiently high (see Figure 5).

\subsection{Spectroscopic sensitivity}

A summary of the spectral line sensitivity for the SLW and SSW bands is shown in Figure 6. The filled square symbols show the pre-launch predictions of the SPIRE sensitivity model ${ }^{20}$. The dashed lines show the sensitivity estimates following the commissioning and PV phases ${ }^{2,6}$. The solid line depicts the best sensitivity values as determined from all of the dark sky observations obtained throughout the mission ${ }^{13}$, using the latest data reduction in HIPE v12. Figure 6 also shows that the sensitivity achieved in flight exceeds the pre-launch estimates by a significant factor. This improvement is due to several factors ${ }^{13}$ : the low telescope background, the fact that the SCAL source is not required to null the interferometer modulation at zero optical path difference (ZPD), the lower operating temperature of the bolometers, and the adoption of a conservatism factor that was applied to the modeled sensitivities to account for various uncertainties in the sensitivity model.

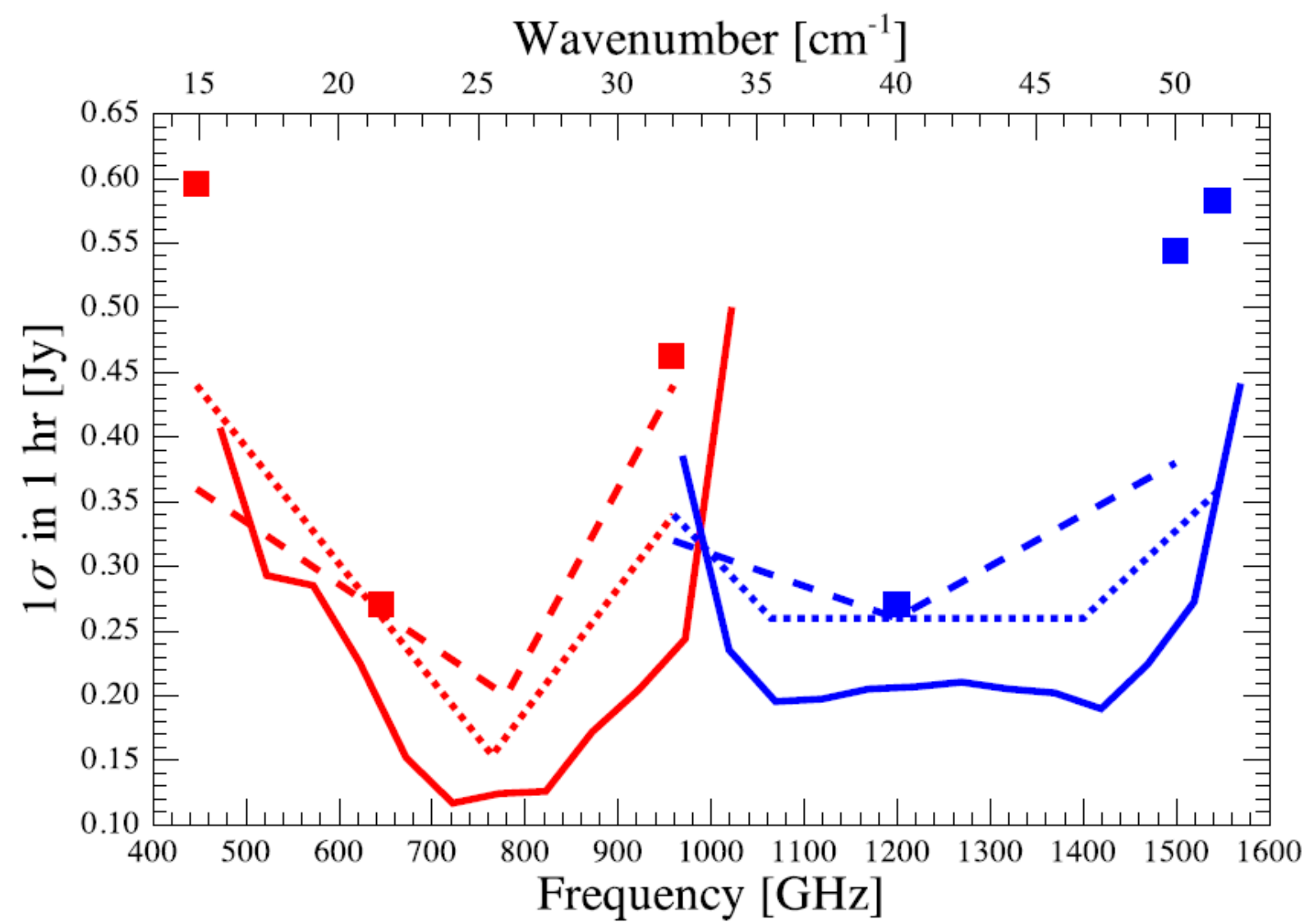

Figure 6. Sensitivity of the SPIRE iFTS. Prelaunch estimates (squares), values used in version 1 (dots) and 2 (dashes) of the Herschel Observation Planning Tool $\left(\mathrm{HSpot}^{21}\right.$ ), and values derived from multiple observations of dark sky, Uranus and Ceres (solid). 
Figure 7 illustrates how the noise integrates down as the square root of the number of scans for the centre and first ring of bolometers in the SLW array and the centre and first two rings of bolometers in the SSW arrays ${ }^{16}$, which correspond to the unvignetted FOV. This figure also serves to show that there is good agreement in the sensitivity across the arrays

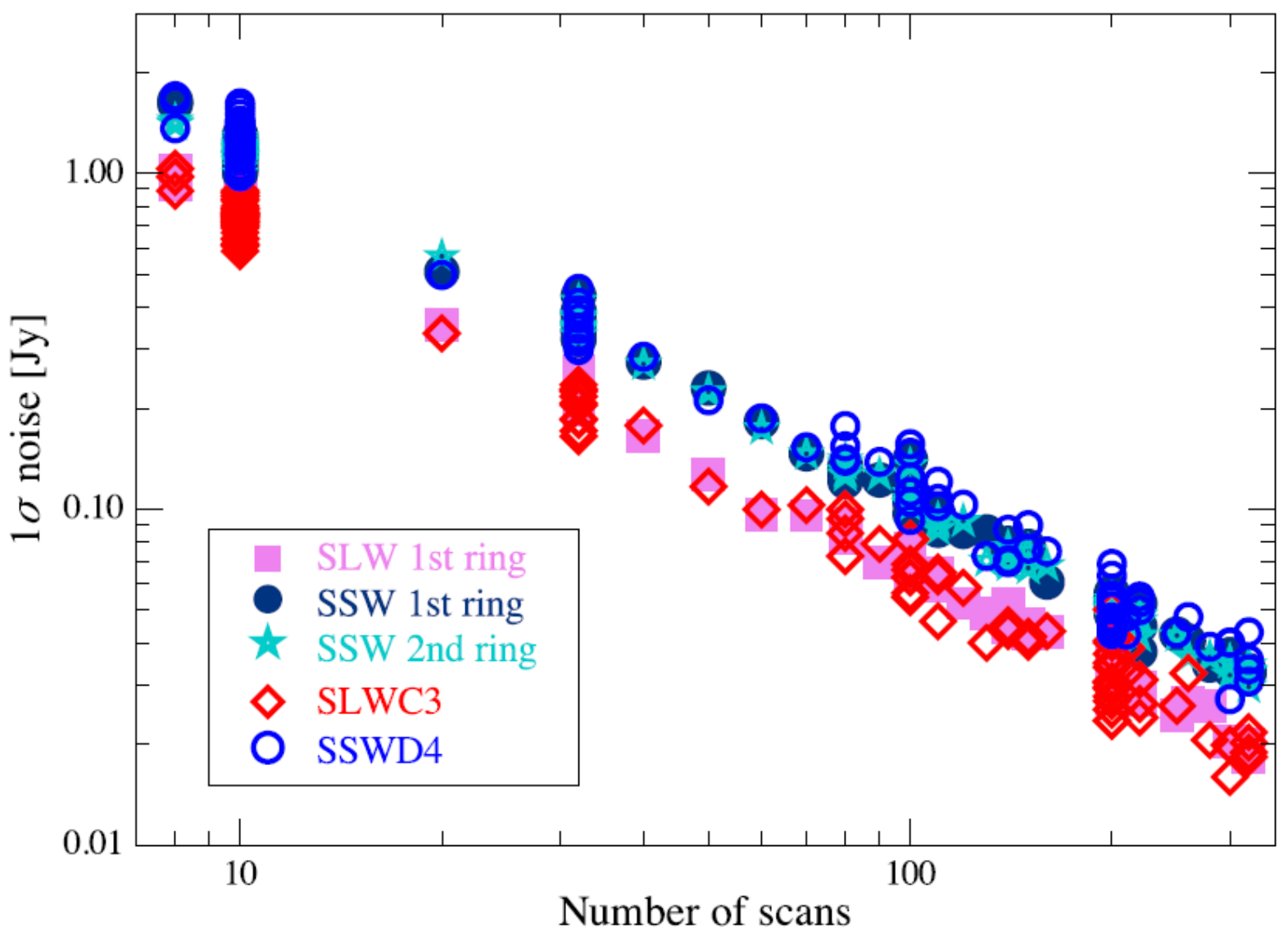

Figure 7. Average noise for dark sky observations as a function of the number of scans, N, for SSW and SLW bolometers (red and blue, respectively). The noise integrates down as $1 / \sqrt{ } \mathrm{N}$ for all bolometers, out to the maximum number of scans obtained for any standard observation at high resolution (320).

\subsection{Beam profile}

The broad spectral coverage of the SLW and SSW bands precludes the use of single-moded feedhorns and results in a complex wavelength dependent spatial illumination of the telescope pupil by the different modes that couple to the conical feedhorns. To interpret correctly the spatial-spectral information conveyed in the measured spectra, however, requires a detailed knowledge of the wavelength dependence of the spectrometer beams. The beam profiles were determined from medium resolution spectra of Neptune, which approximates a point source, as the planet was stepped across the focal plane. A linear superposition of Hermite-Gauss functions, which form a complete set of solutions to the paraxial Helmholtz equation, was fitted to the resulting 3-D spectral-spatial data cube. Details of the fitting procedure and results are described elsewhere ${ }^{22}$. Figure 8 shows the contributions from the $1^{\text {st }}$ and $2^{\text {nd }}$ order modes to the measured beam profile for SLWC3, for a wavelength of $317.5 \mu \mathrm{m}$.

By extending the analysis to each wavelength in the 3-D spatial-spectral data cube the wavelength dependent beam profile can be derived. Figure 9 compares the FWHM of the reconstructed beam to that expected from diffraction theory for the central bolometers in the SLW and SSW arrays. The long-wavelength end of each SPIRE iFTS band agrees well with diffraction theory as expected since the beam is single-moded in these regions. However, the beam shows an increasing deviation from diffraction theory as the number of modes increases. Unfortunately, there was insufficient time to scan Neptune across all of the bolometers. A series of cross scans of the planet across the arrays, however, has 
allowed us to examine the beam profile of a few selected off-centre bolometers. The results show that all of these bolometers exhibit a similar mode structure allowing a representative profile to be adopted.

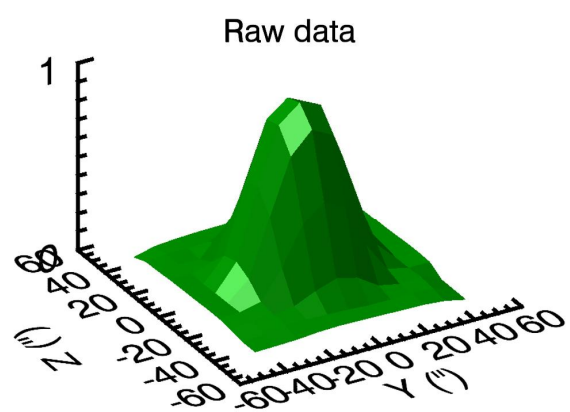

Zeroth order

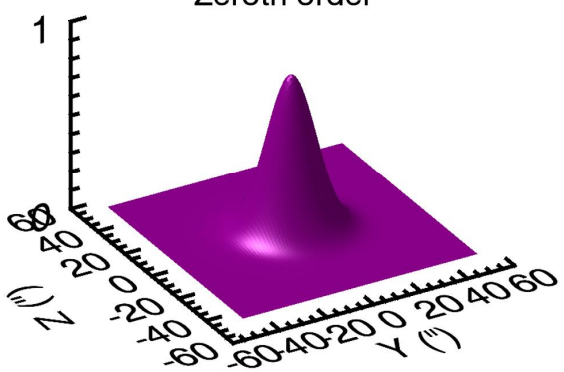

Fitted data

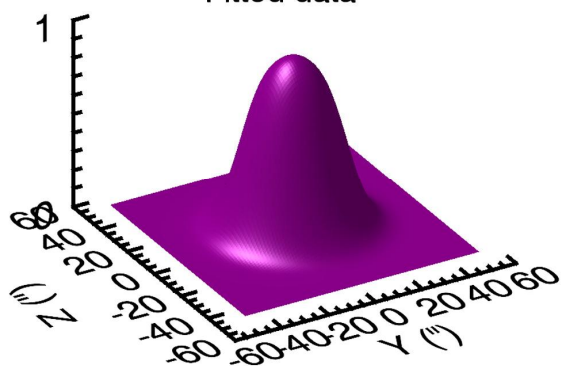

1st order

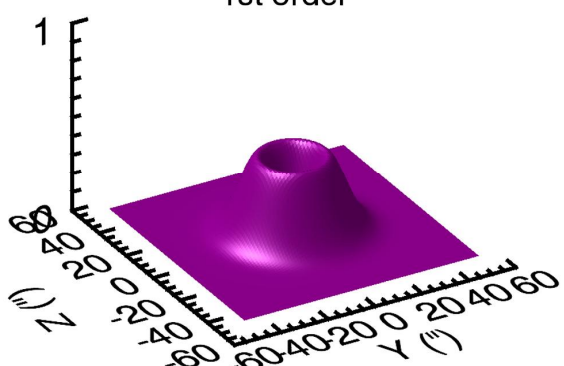

Difference

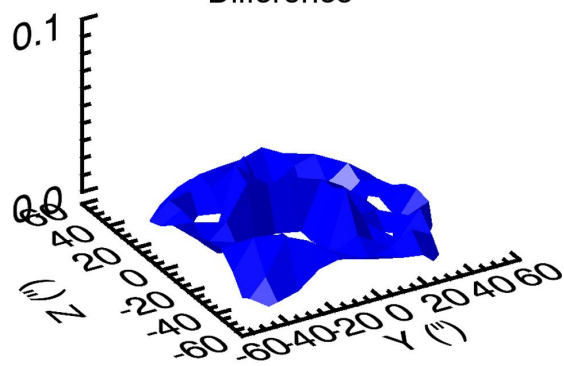

2nd order

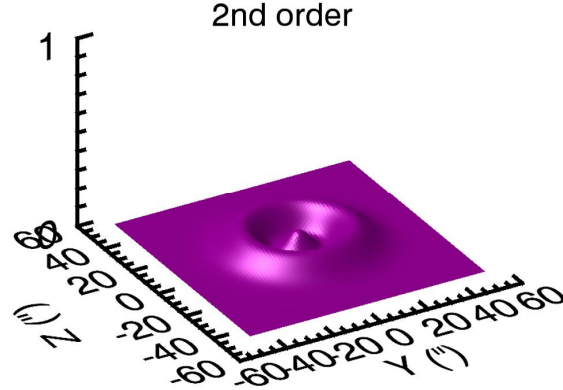

Figure 8. Surface plots obtained from fitting the Neptune 3-D spatial-spectral data cube at a wavelength of $317.5 \mu \mathrm{m}$ (944 GHz). The top row shows the raw data (left), fitted data (centre) and the difference (right; scaled by a factor of 10). The bottom row shows a decomposition of the fitted data (top centre) into the first three Hermite-Gauss modes ${ }^{22}$. The spatial axes are in arcsecs.

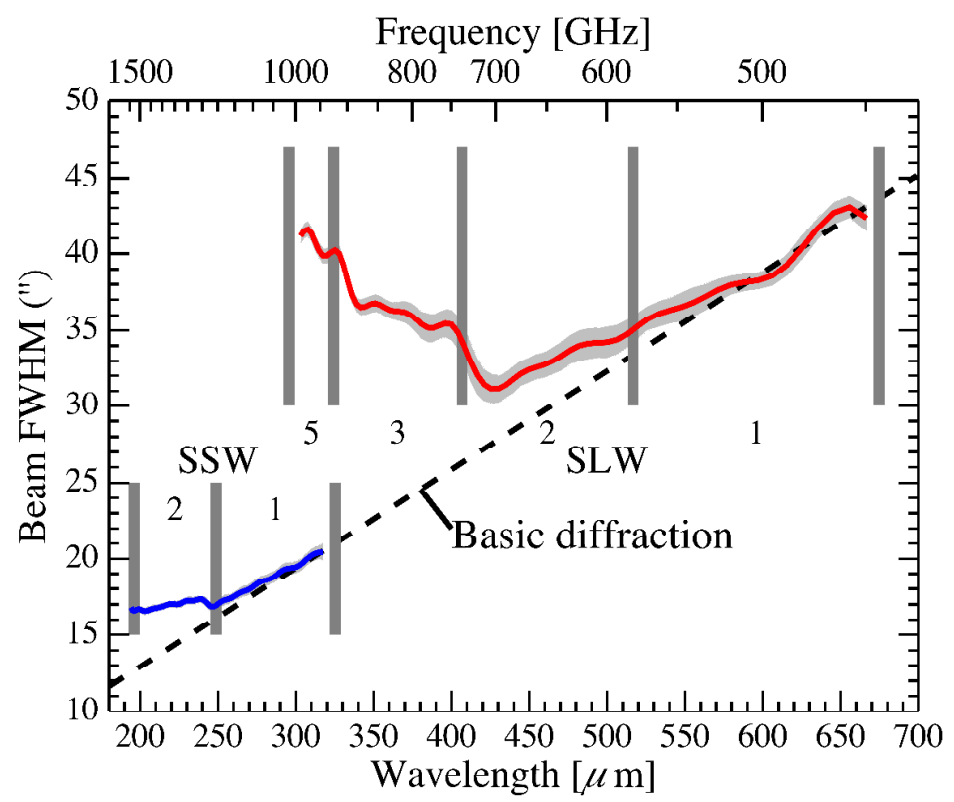

Figure 9. The measured FWHM of the central bolometers SLWC3 (red) and SSWD4 (blue) in arcsecs compared with diffraction theory (dashed line). The gray band represents the $3 \sigma$ errors in the measured FWHM. The vertical lines indicate the cut-on wavelengths for the different feed-horn modes. The expected numbers of modes present in these regions are also indicated ${ }^{22}$.

\subsection{Pointing effects}

Spacecraft pointing variations, expressed in terms of absolute or relative pointing errors (APE and RPE), are potentially troublesome for a Fourier spectrometer as they not only introduce calibration uncertainties, but can also generate spectral artefacts, which result in an additional source of error in retrieved spectral parameters. The APE of Herschel 
observations varied between 1 and 2 arcsecs throughout the mission, with significant improvement down to 0.9 arcsecs for observations later in the mission ${ }^{23}$. Repeated observations of calibration targets, such as AFGL618, throughout the mission, however, yield integrated line intensities that vary by less than $4 \%$, which confirms that the APE is not a dominant source of error ${ }^{13,24}$; this is not unexpected when the APE is compared with the SSW and SLW beamwidths shown in Figure 9. The RPE is a measure of the stability of the telescope once commanded to a given sky position and has been determined to be less than $0.2 \operatorname{arcsecs}^{23}$. While at first glance this figure may seem insignificant, any modulation in the interferogram signal produced by pointing variations, upon Fourier transformation, produces spectral features which cannot be differentiated from those attributed to the incident radiation field. In general, modulation due to RPE manifests itself as a variation in the interferogram baseline, which is proportional to the integrated power reaching the detector. Although the low emissivity primary and secondary mirrors of the Herschel telescope are passively cooled to $\sim 85 \mathrm{~K}$, their emission dominates the measured interferogram signal in all but observations of the brightest astronomical sources ${ }^{10}$. Since this telescope emission is independent of RPE, the effects of the latter are only expected to manifest themselves when observing bright sources whose flux is comparable to that from the telescope.

To illustrate the effects of RPE, the left panel of Figure 10 shows an interferogram of Uranus obtained with the central bolometer of the SSW band, together with a magnified region (inset) in which 22 interferograms from the same observation (OBSID 1342197472) have been overplotted. Although their magnitude is $~ 0.25 \%$ of the peak-to-peak value of the interferogram at zero path difference, baseline variations are still clearly discernable. The right panel of Figure 10 shows the baseline variation of the $11^{\text {th }}$ interferogram from the set of 22 (inset) as a function of the pointing offset in RA and Dec. It can be seen that the drop in signal around $6 \mathrm{~cm}$ OPD corresponds to pointing coordinates whose locus lies to the left of the main group. The interferogram baseline increases around $8 \mathrm{~cm}$ OPD corresponding to pointing coordinates that lie to the upper right of the main group. The fact that pointing variations of $\sim 0.2 \operatorname{arcsec}$ are detectable in the interferogram when observed with the SSW beam of $\sim 17$ arcsec (Figure 9) further demonstrates the sensitivity of the SPIRE instrument.
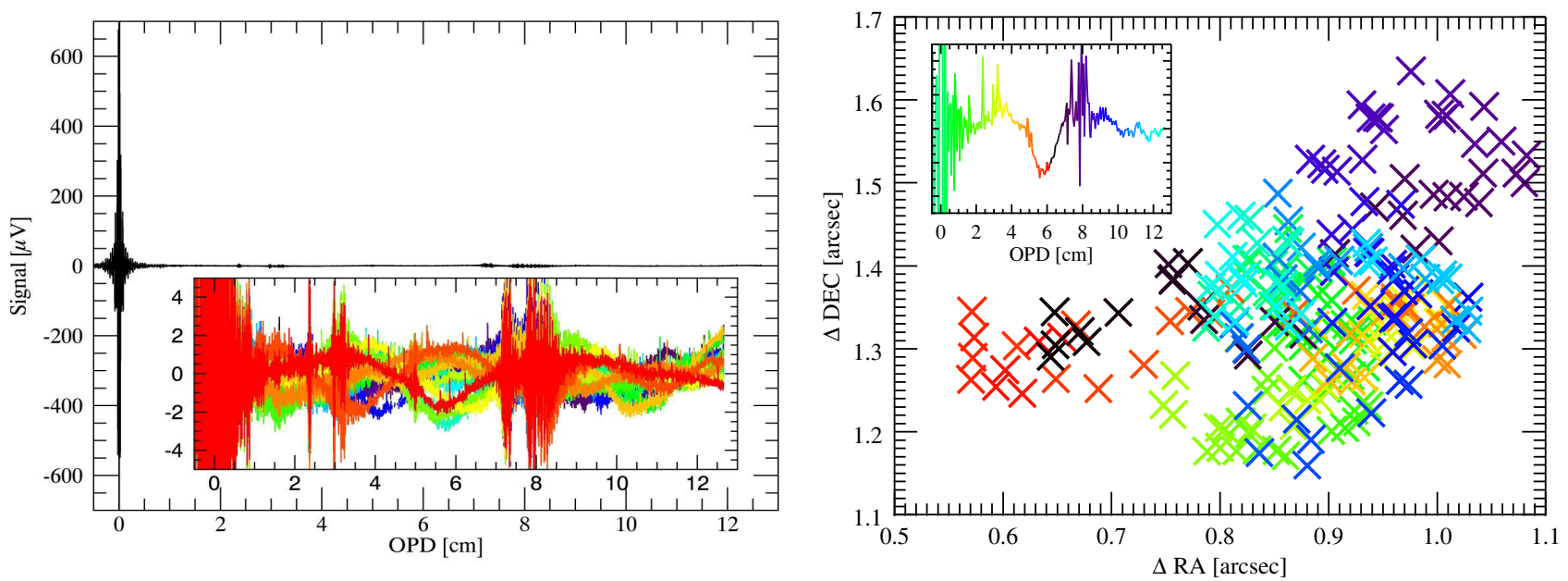

Figure 10. (Left) Interferogram of Uranus measured with the central bolometer (SSWD4) of the SSW array. The inset shows the baseline variation in the 22 Uranus interferograms. (Right) Coloured data points illustrate the OPD correlation of the pointing offset (main plot) with the interferogram baseline variation (inset) (see text for details). The offset from the origin in the plot is a result of the abscissa denoting the RPE offset about the APE.

If flux is lost from one bolometer due to a change in pointing it is reasonable to expect to see an increase on another. In the ideal case the lost signal can be reconstructed from the nearest neighbors, however, the presence of, and variations in interference fringes that are ubiquitous in interferometers (particularly those operating at long wavelengths) precludes such an approach. To illustrate this effect, Figure 11 shows the interferograms out to the first ring of SSW bolometers corresponding to the Uranus observation in Figure 10. It can be seen that the drop in signal at around $6 \mathrm{~cm}$ OPD in the central bolometer (SSWD4) correlates with a slight increase in the upper right bolometer (SSWC3). However, it is also evident that the interference fringes seen in these plots vary from one detector to another. 
The effects of pointing variations on the interferograms measured with the Herschel/SPIRE iFTS are only evident in the SSW band and only when observing the brightest astronomical sources. It should be noted, however, that future far infrared space astronomy missions will employ actively cooled telescope mirrors operating at $\sim 6 \mathrm{~K}$ (e.g. SPICA ${ }^{25}$, FIRI $^{26}$ ) and achieve sensitivities at least two orders of magnitude greater than Herschel. With essentially zero emission from the telescope itself, interferogram modulation due to RPE has the potential to become a dominant noise term. Since a posteriori correction of pointing effects is impractical, this places a stringent requirement on the RPE of these missions.
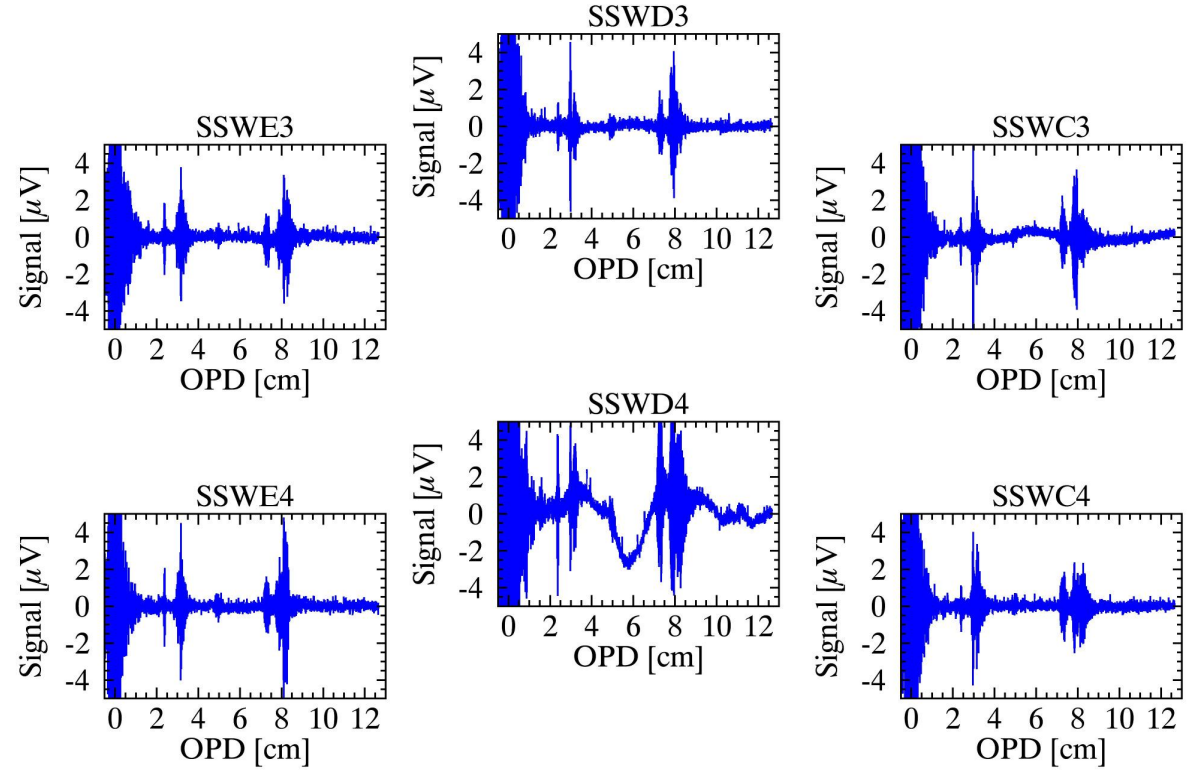

Figure 11. Interferograms for the central and first ring of bolometers in the SSW array corresponding to the measurements of Uranus shown in Figure 10 (bolometer SSWD5 is non-functional). The drop in signal at $6 \mathrm{~cm}$ OPD in SSWD4 corresponds to an increase in SSWC3. Interference fringes at around 3 and $8 \mathrm{~cm}$ OPD resulting from standing waves within the interferometer are evident in each bolometer, however, subtle differences in their characteristics preclude reconstruction of the signal lost due to pointing variations.

\section{IMAGING SPECTROSCOPY}

\subsection{Impact of beam profile}

As mentioned above the two bands of the SPIRE iFTS were designed to have a small overlap region to simplify crosscalibration. If the source under study is point-like or extended ${ }^{13}$, SPIRE spectra can be calibrated, under the assumption that the telescope is well pointed. The different sizes and profiles of the beams at the overlap region of the two bands shown in Figure 9, however, result in the calibration being dependent on source size when the latter falls in between the point and extended regimes. In particular, a large discontinuity and a difference in slope between the SLW and SSW spectra in the overlap region is an indicator that the source size is neither point-like nor fully extended. In such cases neither calibration schema in the nominal data processing pipeline is valid. To illustrate this phenomenon, Figure 12 shows the impact of assuming point- or extended-source calibration for an instrument similar to the SPIRE iFTS when the source is extended (top row), semi-extended (middle row) or point-like (bottom row). In this example, for simplicity, it is assumed that the instrument has a constant beam size of $35 \operatorname{arcsec}$ and $19 \operatorname{arcsec}$ in the SLW and SSW bands, respectively. In each case, the left and right columns show the spectra produced when using the extended- or pointsource calibration schema, respectively. In the top row, the source size has been chosen to be extended for both the SLW and SSW beams, and, as expected the extended source calibration schema yields a continuous spectrum. If these data were calibrated assuming the point source calibration schema, a large discontinuity is seen (top right). In the centre row, the source size has been chosen so that it is extended to the SSW beam yet point-like to the SLW beam. It would thus be necessary to use extended source calibration for SSW and point source calibration for SLW. Without knowledge of the source size, however, discontinuities are present in either calibration schema. Finally, in the bottom row the source has been chosen to be point-like to both the SLW and SSW beams, and the point-source calibration schema produces a continuous spectrum while the extend source calibration introduces a discontinuity as expected. It can be seen from Figure 12 that the nature of the discontinuity provides important information on the source structure, under the assumption that the source is well pointed. It has been shown, however, that the discontinuity is degenerate with respect to telescope pointing errors ${ }^{24}$, thus care must be taken when interpreting the measurements. 

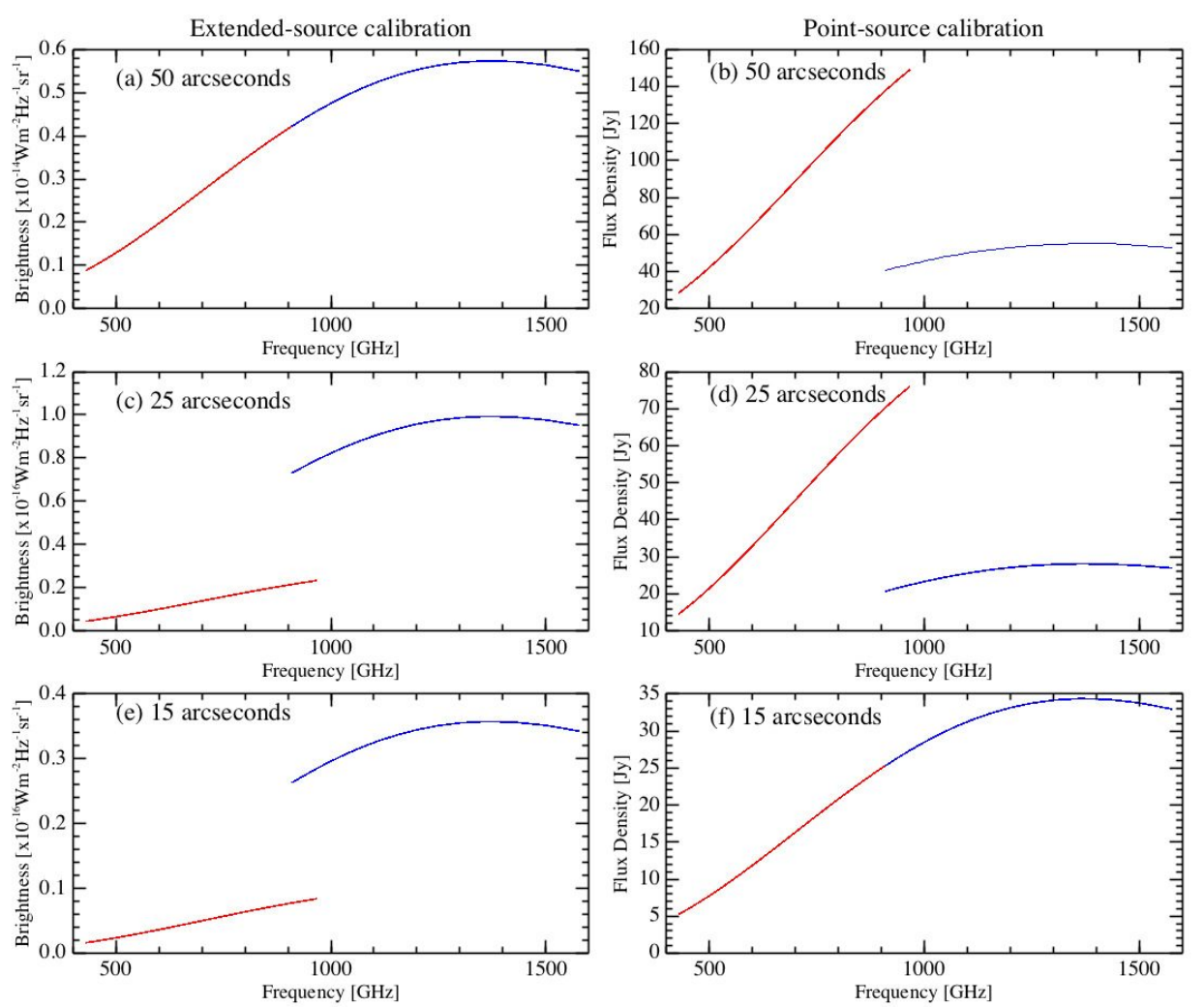

Figure 12. Comparison of the extended source calibration schema (left column) and point source calibration schema (right column) applied to model sources that are: extended (top row), semi-extended (middle row) and point-like (bottom row). See text for details.
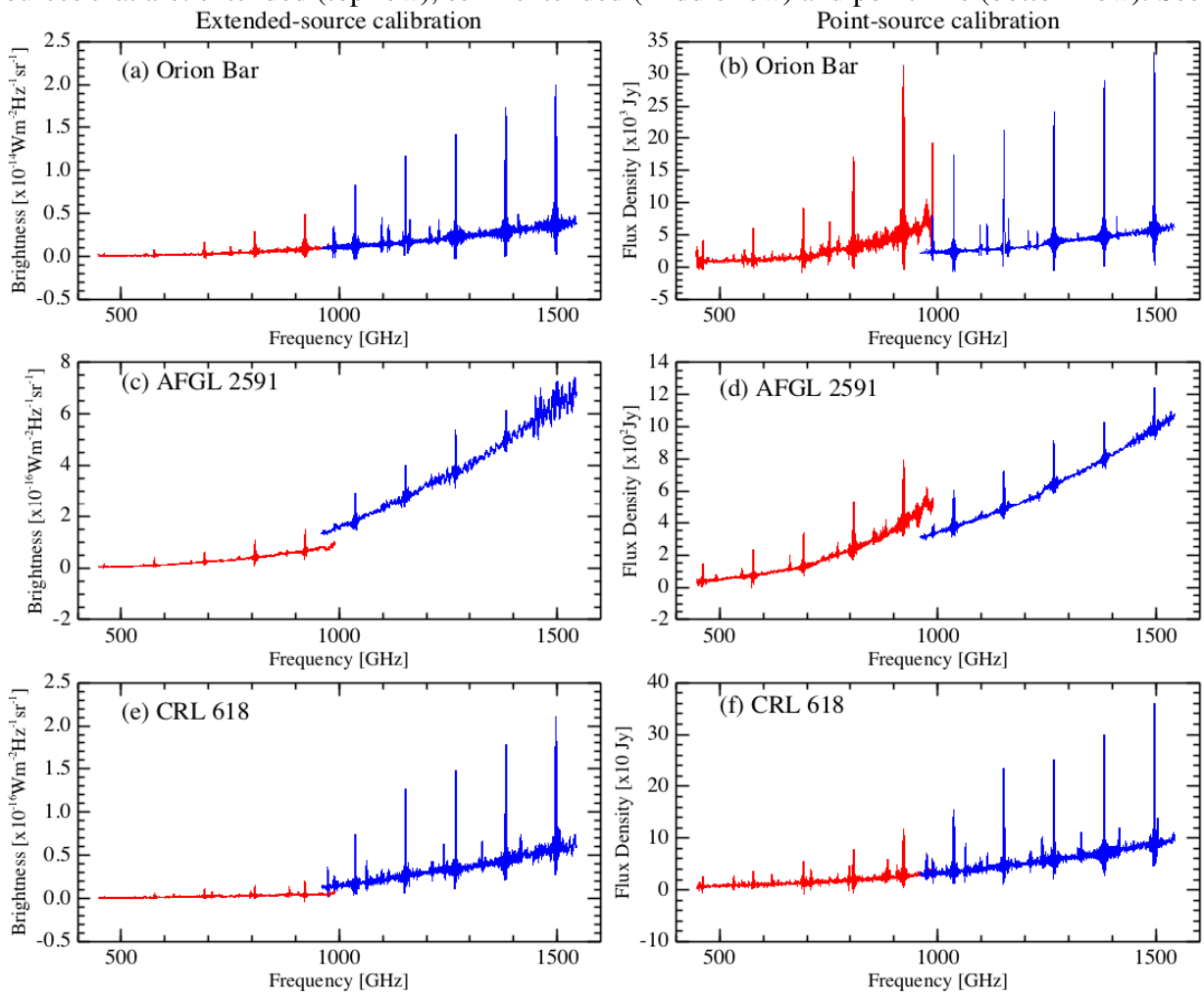

Figure 13. SPIRE iFTS observations of sources with decreasing source size (top to bottom) calibrated using extended source calibration schema (left column) and point source calibration schema (right column). See text for details. 


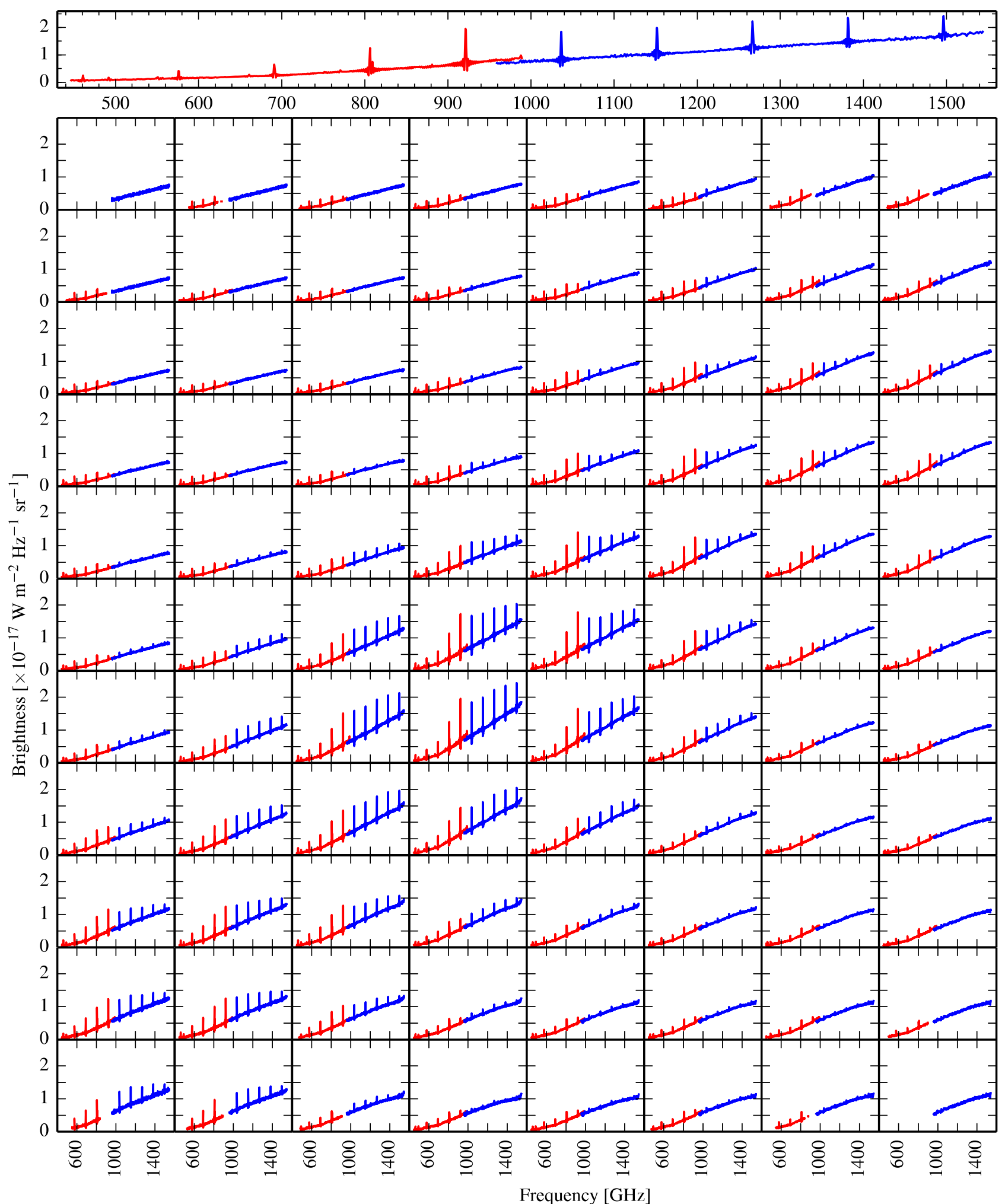

Figure 14: Mosaic of spectral cube resulting from the spatially fully sampled mapping observation of the region around Elias 29 , after convolution to an effective 43 arcsec FWHM beam. SLW spectra are shown in red, SSW spectra in blue. Each pixel is 17.5 by 17.5 arcsecs. RA increases from right to left; Declination increases from bottom to top. The wide top panel shows the spectrum from the pixel with the brightest emission in the map. The spatial variation in line emission from the CO ladder can be clearly seen ${ }^{27}$. 
The theoretical examples shown in Figure 12 can be compared directly with SPIRE iFTS observations of extended, semi-extended and point-like astronomical sources. Figure 13 presents spectra of three sources of decreasing size, which have been processed using both the extended and point source calibration schema. The top row shows the spectra for the Orion bar, a source known to be extended ${ }^{15}$. A reasonable match between the SSW and SLW spectra in the overlap region is obtained by applying extended-source calibration; applying point-source calibration leads to lower flux densities for the SSW band (Figure 12, top right). The middle row shows the spectra of a semi-extended source, AFGL $2591^{28}$. Here neither of the two calibration schemata are valid, a result which confirms the semi-extended nature of the source, (analogous to the middle row of Figure 12). Finally, the bottom row shows the spectra for a point source, CRL $618^{29}$. In this case applying point-source calibration produces a reasonable match between the SSW and SLW spectra, applying extended-source calibration leads to lower flux densities for the SLW band (Figure 12, bottom left). Since most sources observed by SPIRE are semi-extended, a method to correct for source size has been developed ${ }^{30}$ and is implemented in HIPE.

\subsection{Example: mapping observation of a resolved galactic source.}

To illustrate the information content of the broad spectral coverage of the SPIRE iFTS, Figure 14 shows the spatialspectral data cube resulting from a single 3-hour observation of the region around the protostar Elias 29, part of the 'evolution of interstellar dust' key program ${ }^{31}$. The data were processed with the standard HIPE pipeline, modified to project spectra from both bands onto an identical spatial (RA/Dec) world coordinate system ${ }^{27,32}$. Using the beam profile information in Figure 9, the data cube has been convolved in the spatial dimensions with a frequency-dependent Gaussian kernel to convert each frequency slice to a common beam size of 43 ". This approach results in the loss of spatial resolution at the shorter wavelengths, but allows for a quick assessment of the source region under study. It is clear from Figure 14 that the low J CO lines, which predominantly trace cold gas, are seen across the image, while the high J CO lines, which trace hot gas, emanate from a more confined region.

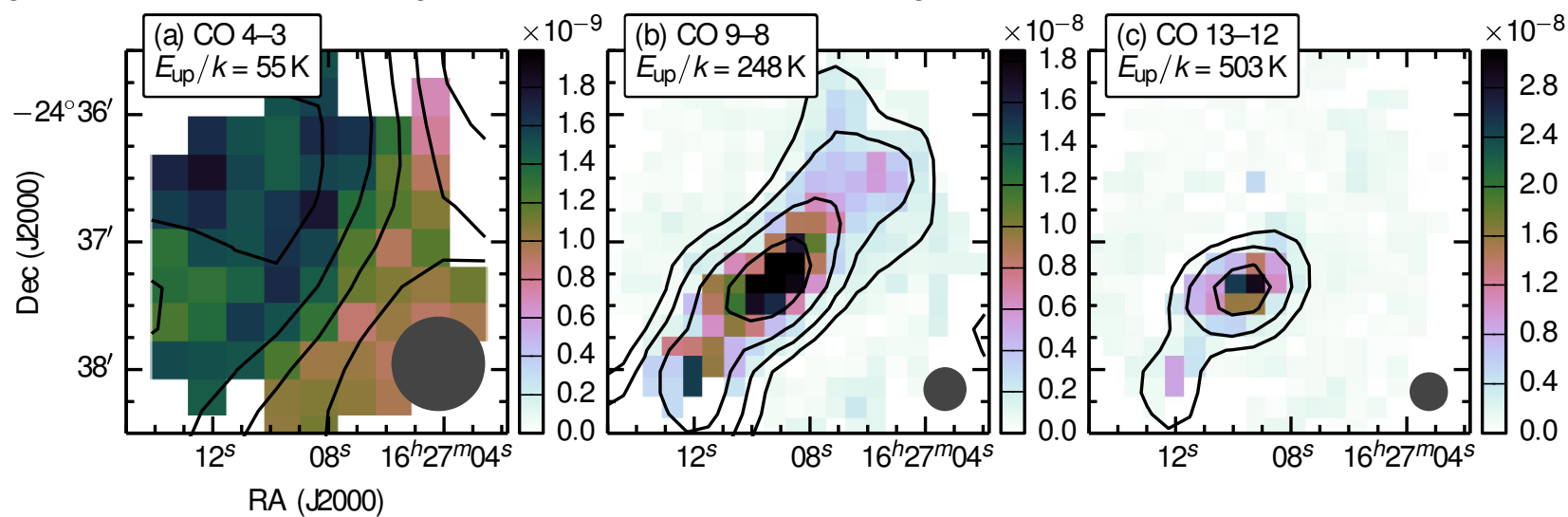

Figure 15: Integrated line intensity maps of three of the ten CO lines covered in the SPIRE iFTS spectral bands. Line intensities, in units of $\mathrm{W} \mathrm{m}^{-2} \mathrm{~Hz}^{-1} \mathrm{sr}^{-1}$ (color bars), are extracted from an unconvolved, fully sampled observation toward the protostellar object Elias $29^{33}$. The temperature of the upper level energy $\left(E_{\mathrm{up}} / \mathrm{k}\right)$ is indicated to illustrate the different physical conditions probed by each transition. The filled circle at the bottom right corner of each panel shows the beam FWHM at the relevant frequency ${ }^{22}$.

To retain the native spatial resolution of the observations shown in Figure 14 the individual spectral lines in the unconvolved data cube can be fitted with a sinc function (shown to be a good approximation to the ILS, see section 2.2), together with a low order polynomial to account for the underlying dust continuum emission. Figure 15 shows integrated line emission maps of three $\mathrm{CO}$ transitions whose morphologies trace different physical components of the interstellar medium around the protostar Elias 29. Lower transitions of CO trace cooler, more tenuous gas, whereas higher transitions trace warm, dense gas. The right panel (c) shows a compact emission peak in the CO 13-12 line at the position of the protostellar envelope, with an embedded, unresolved protoplanetary disk. This observation is consistent with the warmest and densest material being clustered around the centrally heated dense accretion envelope. Panel (b), in the CO 9-8 line, reveals the SE-NW elongated ridge, known to harbor several other star-forming cores. The morphology of the ridge in this map agrees well with independent observations of, for example, far-infrared and submillimetre dust continuum and $\mathrm{HCO}^{+} 3-2$ line emission ${ }^{27,33}$. Finally, panel (a) shows a spatially flat distribution of the $\mathrm{CO} 4-3$ line, which is consistent with spectrally resolved submillimetre observations of lower energy transitions of CO species and carbon sulfide (CS), believed to be located in the foreground of the star-forming complex ${ }^{33}$. The SPIRE FTS maps of 
[CI] fine structure emission (not shown here) also show flat, extended emission consistent with an extended, relatively low-density foreground cloud. These results serve to illustrate the power of the SPIRE iFTS, which by virtue of its large instantaneous FOV and broad spectral bandwidth, probes different physical components in a single observation.

\section{CONCLUSIONS - LESSONS LEARNED}

The performance of the SPIRE imaging Fourier transform spectrometer matched or exceeded pre-launch expectations. In particular, measurements of the spectral range, instrumental line shape, wavelength accuracy, beam profile and sensitivity are in close agreement with either ground-based measurements, or where these were not available, theoretical models. The current spectrometer pipeline produces calibrated spectra of high quality. The use of feedhorns, necessary to control stray light from reaching the sensitive detectors, resulted in a complex wavelength dependent beam profile. Although a telescope simulator was available during the preflight test campaigns, and was used to characterize the SPIRE photometer beam profiles, there was insufficient time to explore the nature of the beam for the SPIRE iFTS; such measurements would have been very useful. Spectra of Neptune obtained as the planet was raster scanned across the FOV provided a unique spatial-spectral data cube that enabled a thorough investigation of the beam profile, albeit only for the central detectors of each array. Although these calibration measurements were time consuming, the knowledge of the beam profile that they provide is essential for the correct interpretation of the spatial-spectral information conveyed in the measured spectra. The effects of spacecraft pointing errors on data collected by the SPIRE iFTS, while only evident when observing the brightest astronomical sources in the SSW band, serve to illustrate the challenges that lie ahead for the next generation of far-infrared space astronomy missions that employ imaging Fourier transform spectrometers. With telescope mirrors actively cooled to cryogenic temperatures $(\sim 6 \mathrm{~K})$ future missions such as SPICA ${ }^{25}$ and FIRI ${ }^{26}$ will employ detectors with at least two orders of magnitude better sensitivity than those flown on Herschel. By necessity these detectors will be feedhorn coupled to meet the challenging stray light requirements. With essentially zero emission from the telescope itself, interferogram modulation due to spacecraft pointing errors will become far more severe and has the potential to become a dominant noise term. Since a posteriori correction of pointing effects is impractical, this places a stringent requirement on the telescope RPE for future missions.

Notwithstanding these issues, the spectra produced by the SPIRE iFTS are of the highest quality and will allow astronomers to address leading questions in modern astrophysics for years to come.

\section{ACKNOWLEDGMENTS}

SPIRE has been developed by a consortium of institutes led by Cardiff Univ. (UK) and including Univ. Lethbridge (Canada); NAOC (China); CEA, LAM (France); IFSI, Univ. Padua (Italy); IAC (Spain); Stockholm Observatory (Sweden); Imperial College London, RAL, UCL-MSSL, UKATC, Univ. Sussex (UK); Caltech, JPL, NHSC, Univ. Colorado (USA). This development has been supported by national funding agencies: CSA (Canada); NAOC (China); CEA, CNES, CNRS (France); ASI (Italy); MCINN (Spain); SNSB (Sweden); STFC (UK); and NASA (USA). DAN and LDS acknowledge support from CFI and NSERC. The authors thank Tanner Heggie, Courteney Hamilton, Adam Lefaivre, and Jeremy Scott for their assistance in the analysis.

\section{REFERENCES}

[1] Pilbratt, G. et al., "Herschel Space Observatory - An ESA facility for far-infrared and submillimetre astronomy," Astronomy and Astrophysics 518, L1 (2010).

[2] Griffin, M.J. et al., "The Herschel-SPIRE instrument and its in-flight performance," Astronomy and Astrophysics, 518, L3 (2010).

[3] Griffin, M., et al., "Herschel-SPIRE: design, ground test results, and predicted performance," Proc. SPIE, 7010, 701006 (2008).

[4] Ade, P.A.R, Hamilton, P.A., Naylor, D.A., "An Absolute Dual Beam Emission Spectrometer," Optical Society of America, FTS topical meeting poster FWE3, Santa Barbara, California (1999).

[5] Ade, P.A.R. et al., "A review of metal mesh filters," Proc. SPIE, 6275, 62750U (2006).

[6] Naylor, D.A., et al., "In-orbit performance of the Herschel/SPIRE imaging Fourier transform spectrometer," Proc. SPIE 7731, 29 (2010).

[7] Ott, S., "The Herschel Data Processing System - HIPE and Pipelines - Up and Running Since the Start of the Mission," Astronomical Data Analysis Software and Systems XIX, eds. Mizumoto, Y., Morita, K.-I. and Ohishi, M., 434, 139 (2010). 
[8] De Graauw, T. et al., "The Heterodyne Instrument for the Far Infrared (HIFI) on the Herschel Space Observatory," Astron. Astrophys. 518, L6 (2010).

[9] Poglitsch, A. et al. 2010, "The Photodetector Array Camera and Spectrometer (PACS) on the Herschel Space Observatory," Astron. Astrophys. 518, L2 (2010).

[10] Hopwood, R. et al., "Herschel SPIRE FTS telescope model correction," Experimental Astronomy, Online First (2013).

[11] Lu, N. et al., "Herschel SPIRE Fourier transform spectrometer: calibration of its bright-source mode," Experimental Astronomy, Online First (2013).

[12] Fulton, T. et al., "Herschel SPIRE FTS relative spectral response calibration," Experimental Astronomy, Online First (2014).

[13] Swinyard, B.M, et al., "Calibration of the Herschel SPIRE Fourier Transform Spectrometer," Monthly Notices of the Royal Astronomical Society 440, 3658-3674 (2014).

[14] Naylor, D. A. \& Tahic, M.K., “Apodizing functions for Fourier transform spectroscopy," J. Opt. Soc. Am. A, 24, 3644-3648,(2007).

[15] Habart, E. et al., "SPIRE spectroscopy of the prototypical Orion Bar photodissociation region," Astronomy and Astrophysics 518, L116 (2010).

[16] Hopwood, R. et al., "Routine calibration programme of the Herschel SPIRE Fourier transform spectrometer," in prep.

[17] Davis, S. P., Abrams, M. C. \& Brault, J. W., "Fourier Transform Spectrometry," Academic Press (2001).

[18] Spencer, L.D., Naylor, D.A. and Swinyard, B.M., "Performance evaluation of the Herschel/SPIRE imaging Fourier transform spectrometer through ground-based measurements," Meas. Sci. Technol. 21, 5601 (2010).

[19] Fulton, T.R., et al., "The data processing pipelines for the Herschel/SPIRE imaging Fourier transform spectrometer," Proc. SPIE 7731, 99 (2010).

[20] SPIRE Observers' Manual, HERSCHEL-HSC-DOC-0789, version 2.0, May 2010 available at the ESA Science Centre web site: http://herschel.esac.esa.int/Docs/SPIRE/html/spire_om.html

[21] HSpot is a joint development by the Herschel Science Ground Segment Consortium, consisting of ESA, the NASA Herschel Science Center, and the HIFI, PACS and SPIRE consortia. $\mathrm{ftp}: / / \mathrm{ftp}$.sciops.esa.int/pub/hspot/HSpot_download.html

[22] Makiwa, G., et al., "Beam profile for the Herschel-SPIRE Fourier transform spectrometer," Applied Optics 52, 3864-3875 (2013).

[23] Sánchez-Portal, M., et al., "The Pointing System of the Herschel Space Observatory: Description, Calibration, Performance and Improvements," Experimental Astronomy (submitted).

[24] Valtchanov, I., Hopwood, R., Polehampton, E.T., et al., "Relative pointing offset analysis of calibration targets with repeated observations with Herschel-SPIRE Fourier-transform spectrometer," Experimental Astronomy, Online First (2013).

[25] Swinyard, B. M. et al., "The space infrared telescope for cosmology and astrophysics: SPICA A joint mission between JAXA and ESA," Experimental Astronomy, 23, 193 (2009).

[26] Helmich, F. and Ivison, R., "FIRI - a Far-Infrared Interferometer," Experimental Astronomy, 23, 245 (2009).

[27] Makiwa, G., "A study of the physics of the interstellar medium using the Herschel-SPIRE instrument," Ph.D. thesis, University of Lethbridge (2014).

[28] Van der Tak, F.F.S., "The Impact of the Massive Young Star GL 2591 on its Circumstellar Material: Temperature, Density, and Velocity Structure," Astrophysical Journal 522, 991 (1999).

[29] Nakashima, J. et al., "Submillimeter Array Observation of the Proto-Planetary Nebula CRL 618 in the CO J = 6-5 Line," Astronomical Journal 134, 2035 (2007).

[30] Wu, R. et al., "Observing extended sources with the Herschel SPIRE Fourier Transform Spectrometer," Astronomy and Astrophysics 556, A116 (2013).

[31] Abergel, A. et al., "Evolution of interstellar dust with Herschel. First results in the photodissociation regions of NGC 7023," Astronomy and Astrophysics, 518, L95 (2010).

[32] Benielli, D. et al., "Herschel SPIRE FTS spectral mapping calibration," Experimental Astronomy, Online First (2014).

[33] Boogert, A.C.A. et al., "The Environment and Nature of the Class I Protostar Elias 29: Molecular Gas Observations and the Location of Ices," Astrophysical Journal 570, 708 (2002). 